\title{
Longitudinal Changes in the Blood Supply and Demand in North-East-Germany 2005-2015
}

\author{
Linda Schönborn $^{a}$ Kerstin Weitmann ${ }^{b}$ Nico Greger ${ }^{c}$ Volker Kiefelc Wolfgang Hoffmann ${ }^{b}$ \\ Andreas Greinacher ${ }^{a}$
}

${ }^{a}$ Institut für Immunologie und Transfusionsmedizin, Universitätsmedizin Greifswald, Greifswald, Germany;

${ }^{\mathrm{b}}$ Institut für Community Medicine, Universitätsmedizin Greifswald, Greifswald, Germany;

${ }^{\mathrm{c}}$ Institut für Transfusionsmedizin, Universitätsmedizin Rostock, Rostock, Germany

\section{Keywords}

Allogeneic blood - Allogeneic transfusion .

Blood donation - Blood safety - Blood supply .

Blood transfusion - Donors - Red blood cell concentrate .

Transfusion . Whole blood . Donor research .

Demographic change

\section{Summary}

Background: Securing future blood supply is a major issue of transfusion safety. In this prospective 10-year longitudinal study we enrolled all blood donation services and hospitals of the federal state Mecklenburg-Western Pomerania. Methods and Results: From 2005 to 2015 (time period with major demographic effects), whole blood donation numbers declined by $18 \%$. In male donors this paralleled the demographic change, while donation rates of females declined $12.4 \%$ more than expected from demography. In parallel, red cell transfusion rates/1,000 population decreased from 2005 to 2015 from 56 to 51 (-8.4\%), primarily due to less transfusions in patients $>60$ years. However, the transfusion demand declined much less than blood donation numbers: $-13.5 \%$ versus $-18 \%$, and the population $>65$ years (highest transfusion demand) will further increase. The key question is whether the decline in transfusion demand observed over the previous years will further continue, hereby compensating for reduced blood donation numbers due to the demographic change. The population structure of Mecklenburg-Western Pomerania reflects all Eastern German federal states, while the Western German federal states will reach similar ratios of age groups $18-64$ years / $\geq 65$ years about 10 years later. Conclusions: Regular monitoring of age- and sex-specific donation and transfusion data is urgently required to allow transfusion services strategic planning for securing future blood supply.

(C) 2017 S. Karger GmbH, Freiburg

\section{Introduction}

In North-East Germany major demographic changes occurred after the German reunification in 1990. The birth rate declined by more than $50 \%$ (fig. 1) and large numbers of young people, especially women, moved to Western federal states for employment.

In Germany blood donors have to be at least 18 years old. Thus, the effects of these abrupt demographic changes since 1990 manifested in the blood donor population from on 2008/2009, leading to a decline of whole blood donations. At the same time, the proportion of the population older than 65 years of age increased as it is generally observed in European countries. About two-thirds of the transfusion demand is required for patients older than 65 years of age due to their higher likelihood for (co-)morbidities requiring blood transfusions. We used this major demographic change to prospectively analyze the impact of demographic changes on the blood supply by a longitudinal study starting in 2005 in the federal state Mecklenburg-Western Pomerania [1-3].

We found that whole blood donations can be reasonably predicted based on future trends of demographic structures, but they are also influenced by changes in donor recruitment activities [2, $3]$. In contrast, the transfusion demand for red blood cell concentrates (RBCs) is more influenced by changes in transfusion practice [2-5]. In recent years all western countries observed a decrease in transfusion demand [4-9]. This is in part driven by the availability of prospective randomized trial data showing that liberal transfusion strategies (maintaining the patient's hemoglobin levels above $10 \mathrm{~g} / \mathrm{dl}$ ) do not seem to be of benefit for patients [10], and has further been enforced by implementation of the 'patient blood management program', which comprises a number of different measurements including optimizing the preoperative hemoglobin level of the patient, reduction of perioperative blood loss, and a restrictive transfusion strategy [11].

In the present study we extend previous analyses of our longitudinal study on blood supply and transfusion demand between the

\section{KARGER}

(c) 2017 S. Karger GmbH, Freiburg

Fax +497614520714 


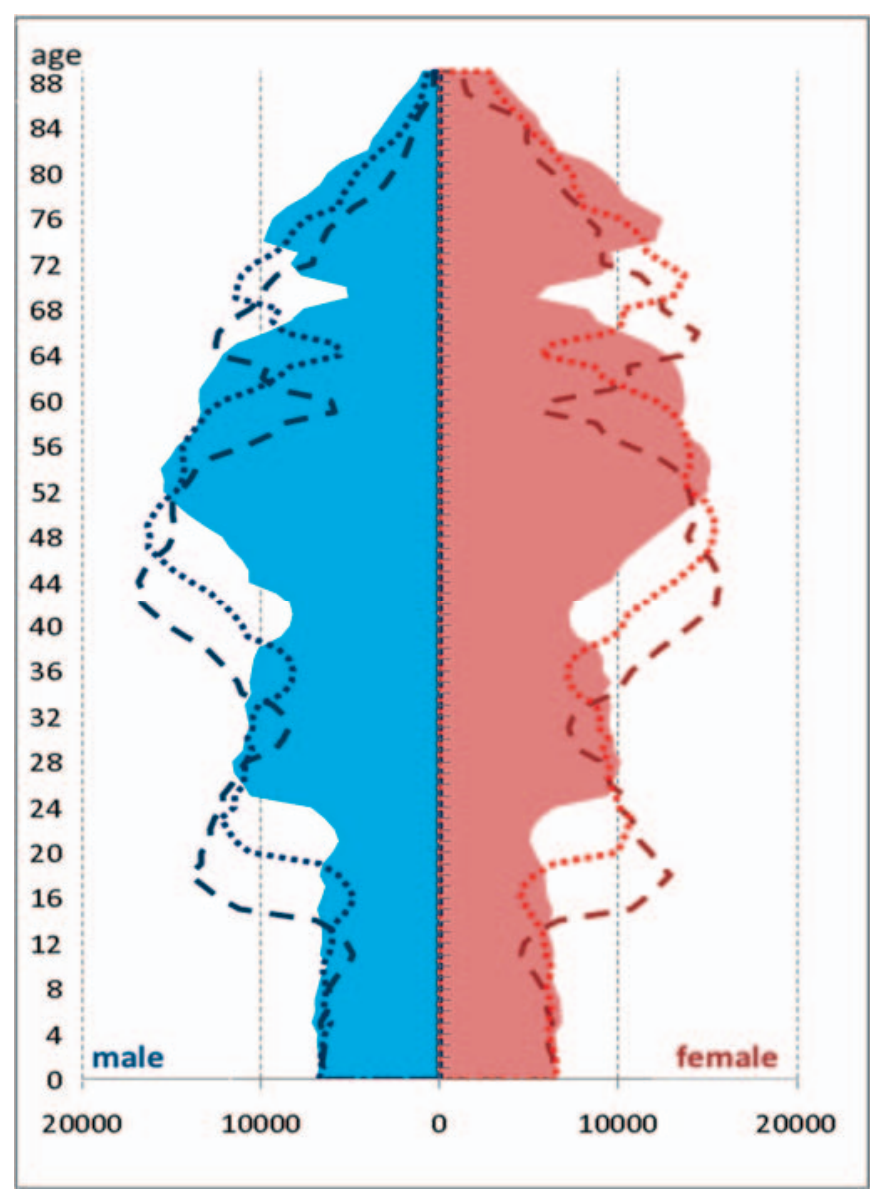

Fig. 1. Population of Mecklenburg-Western Pomerania 2005, 2010, 2015 [12] The population structure reflects the three major events leading to a birth decline in the state: World War II, followed by the baby boomer generation, introduction of hormonal oral contraceptives in the 1970s, and the German reunification in 1990.

years 2005, 2010, and 2015 [1-3] by addressing special aspects of the demographic changes in different parts of Germany as well as age and gender distribution of whole blood donors and $\mathrm{RBC}$ recipients.

\section{Material and Methods}

For each whole blood donation and red blood cell apheresis obtained in the federal state Mecklenburg-Western Pomerania in the years 2005, 2010, and 2015 the following characteristics of the donor were determined: age (or date of birth), sex of the donor, and date of donation. We counted each donated RBC as one event ( $\mathrm{RBC}$ donations by apheresis were counted as $2 \mathrm{RBCs}$ ). The data were provided by the four blood donation services operating in the state (Red Cross blood donation service; blood donation service of the University Hospital Rostock; blood donation service of the University Hospital Greifswald; HAEMA Blutbank (private blood donation service)) for the years 2005, 2010, and 2015.

For each RBC transfused to in-hospital patients in Mecklenburg-Western Pomerania in the years 2005, 2010, and 2015 the following characteristics of the patient were determined: age (or date of birth), sex as well as patient classification (surgical, medical, critically ill / emergency room, pediatric), and date of transfusion. The data were obtained from the 40 hospitals in the state for the years 2005, 2010, and 2015 [1-3]. As pediatric and non-classifiable patients account for less than $3 \%$ of all RBC transfusions these were not analyzed in detail.
The population registry for Mecklenburg-Western Pomerania provided population data in 1-year age groups for females and males for 2005, 2010, and 2015 [12]. These data were used to calculate the gender- and age-specific number of donations and transfusions per 1,000 inhabitants. For comparison of the actual and predicted demographic structure of the federal states of Germany, population data were obtained from the Federal Statistical Office [13-15]. According to the type of data documentation in the hospitals, the recipient data were classified as digital (all data were retrieved from the hospital IT system), partially digital (data were retrieved from laboratory books as well as from the hospital IT system), and non-digital. The latter had to be abstracted manually or were transcribed from paper documentation (e.g. laboratory books) into the study database. Data were double-checked to assure data quality.

The institutional ethics review board of the Universitätsmedizin Greifswald approved the study. In the analyses exclusively anonymous data were used.

\section{Results}

Impact of Demographic Changes on the Potential Donor Population and the Transfusion Recipient Population

Demographic changes lead to an increase in the population group $\geq 65$ years of age and a decrease in the population of the younger age groups. Figure 1 shows the demographic age distribution in Mecklenburg-Western Pomerania in the years 2005, 2010, and 2015. The population $<65$ years of age decreased, while at the same time the age group $\geq 65$ years increased from $19.7 \%$ of the population in 2005 to $23.0 \%$ in 2015 . These changes are highly relevant for transfusion medicine as the majority of whole blood donations was obtained from donors $<65$ years (2005: 99.1\%; 2010: 98.0\%; 2015: 96.7\%), but about two-thirds of RBCs were transfused to patients in the age group $\geq 65$ years (2005: $60.3 \%$; 2010 : $66.2 \%$; 2015: $62.6 \%$ ). Thus the ratio between the populations $18-64$ years and $\geq 65$ years of age strongly determines the balance between blood supply and blood demand.

To put these data into perspective with other regions in Germany, we obtained the data on age distribution of the population for all states in Eastern Germany, all states in Western Germany, and separately for the 'city states' Berlin, Hamburg, and Bremen due to their different population structure. In 2005, the ratio of the population 18-64 years / $\geq 65$ years in Mecklenburg-Western Pomerania was 3.3; in 2010 2.9; and in 2015 2.7; in 2020 it is expected to be 2.3; and will approach 1.7 in 2030 [15]. A similar shift in demographics is noted for all states in Eastern Germany, while the demographic changes are delayed in the Western German states where a ratio of 2.2 will be reached about 10 years later in 2030 (fig. 2) [13, 14].

\section{Development of the Donor Population Focusing on Age Group and Gender Disparities}

As reported [3], within the 10-year study period the absolute number of whole blood donations first increased from 118,419 in 2005 to 132,291 in 2010, likely as a consequence of the activities of a new private blood donation service, but then decreased to 97,045 donations in 2015. This corresponds to an overall decline of $18 \%$ between 2005 and 2015. This effect is mostly related to a decline of blood donations in the age group 18-29 years (2005: 39,199 RBCs; 
Fig. 2. Ratio of population $18-64$ years old / $\geq 65$ years old for Eastern German federal states, Western German federal states, City federal states, and Mecklenburg-Western Pomerania [13]. The ratio between the population in 18- to 64-year age group and the population in the $\geq 65$ year age group decreases in all regions of Germany with a more pronounced and earlier decrease in the Eastern German federal states in whom the changes manifest about 10 years earlier than in the Western German federal states.

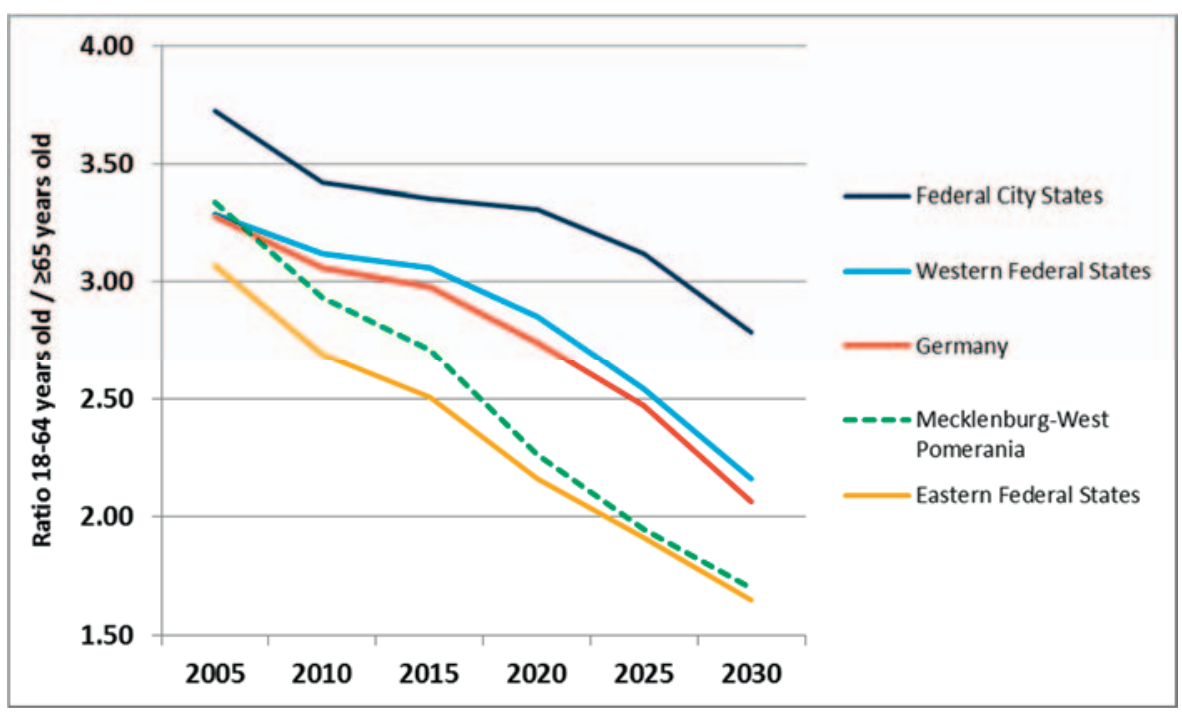

2015: 24,475 RBCs; -37.6\%; fig. 3A) and here especially by a decline of blood donations by young women with $-45.8 \%$ (2005: 17,730 RBCs; 2015: 9,613 RBCs) while donations by young men declined by $-30.8 \%$ (2005: 21,469 RBCs; 2015: 14,862 RBCs). Considerably less pronounced is the decrease of donations obtained from donors 30 years and older. But also here a gender difference is obvious. Donations from male donors declined by $4.0 \%$ and from female donors by $12.9 \%$. All together in $2005,48.0 \%$ of whole blood donations were obtained from female donors, this proportion declined to $46.0 \%$ in 2010 and to $45.0 \%$ in 2015 .

An interesting observation is that the age group with most donations in the age groups $\geq 30$ years shifted in both men and women from $40-44$ years in 2005 (corresponding to $14.1 \%$ of male and $16.7 \%$ of female donations) to $45-49$ years in 2010 (male $13.2 \%$, female $16.1 \%$ ), and to $50-54$ years in 2015 (male $14.0 \%$, female $16.6 \%$ ). This reflects the age shift of the biggest population group consisting of the 'baby boomer' generation (fig. 1). The lowest absolute donation numbers in donors younger than 60 years show a similar shift of age groups: male and female donors had their lowest absolute donation numbers in the age groups 28-32 years (2005), 33-37 years (2010), and 38-42 years (2015). This overlaps with the population decline in this age group known as the 'contraceptive pill gap' of the late 1960s (fig. 1).

In comparison to the absolute numbers of whole blood donations, the donation rates per 1,000 inhabitants are shown in figure $3 \mathrm{~B}$. The relative changes in donation rates were not as pronounced as the absolute changes, especially when the numbers of 2005 and 2015 are compared (95 to 89/1,000 inhabitants). The whole blood donation rate in men remained rather constant with $96 / 1,000$ population in 2005 and 95/1,000 population in 2015. However, it declined in women from $94 / 1,000$ inhabitants in 2005 to $82 / 1,000$ inhabitants in 2015. For men as well as for women the highest donation rates per 1,000 population were found in the age group 18-29 years. For male donors the donation rate in this age group increased from 147 in 2005 to 216 in 2010 and then decreased to $148 / 1,000$ population in 2015 . For female donors, donation rates in this age group (18-29 years) first increased from 141 in 2005 to 164 in 2010 but then remarkably decreased to $109 / 1,000$ inhabitants in 2015 (fig. 3b). Nevertheless the population between 18 and 30 years of age still shows the highest blood donation rate per 1,000 population. The increases in donation rates between 2005 in 2010 are most likely attributed to the activities of a new blood service, which caused a transient increase in blood donation rates.

\section{Age Structure of Whole Blood Donations by Different Blood}

Donation Services

Donors of the four blood donation services have different age structures. The relative and absolute proportion of blood donors per age group of the Red Cross blood donation service and the other blood services (combined) are shown in fig. 4A,B (all blood donations of a single blood donation service correspond to $100 \%$ ). The university-based blood services as well as the private blood service primarily motivated younger blood donors and obtained the highest amount of RBCs from donors in the age group 20-29 years, a pattern which remained constant between 2005 and 2015. In contrast most blood donors of the Red Cross blood donation service were above 30 years of age. Remarkably, considering all blood services, $39.7 \%$ of RBCs were donated by donors $\geq 50$ years of age in 2015.

As shown in figure $4 \mathrm{~B}$ the different blood services obtained largely different absolute numbers of whole blood donations. Most blood donations were obtained by the Red Cross blood donation service. This remained constant over the 10-year study period: 2005: 71.6\%; 2010: 64.4\%; 2015: 64.4\%.

\section{Development of In-Hospital Transfusions by Patient Classification}

The absolute number of in-hospital transfusions was rather constant between 2005 (95,455 RBCs) and 2010 (95,200 RBCs) but decreased to 82,591 RBCs in 2015 , which corresponds to a total reduction of in-hospital transfusions by 13.5\% [3] (table 1). Medical patients required the largest proportion of RBCs, and the percent- 

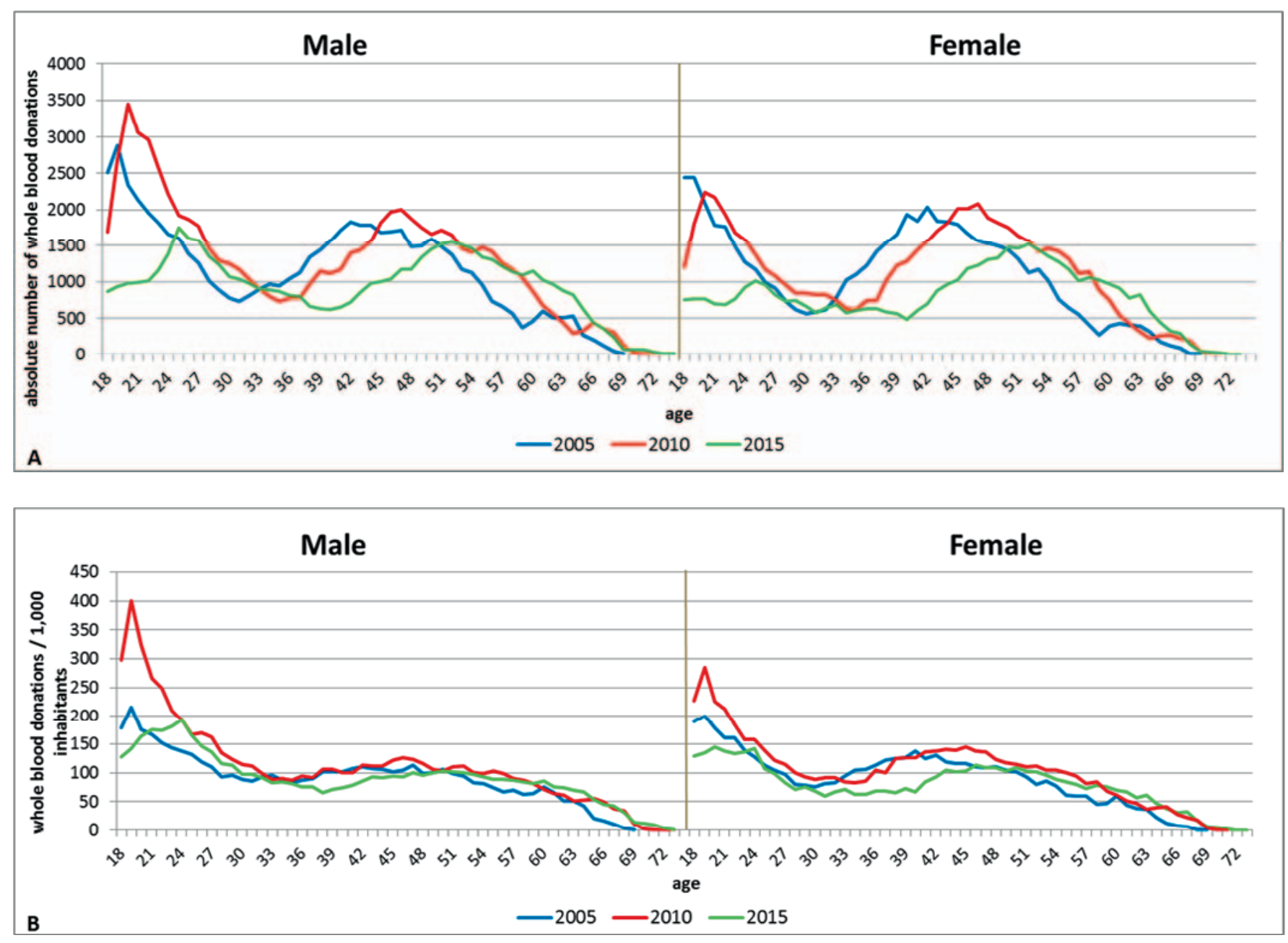

Fig. 3. A Absolute number of whole blood donations by sex. Absolute numbers of whole blood donations decreased in Mecklenburg-Western Pomerania between 2005 and 2015, primarily because of a decline in the younger age groups. In addition the peak of blood donations in the group $>40$ years of age shifts and parallels aging of the baby boomer generation. This indicates that in 10-15 years the blood donors who contribute today a large proportion of whole blood donations will leave the donor pool, without sufficient replacement by younger donors. These effects are similar for male and female donors. B Whole blood donations per 1,000 inhabitants by sex. The change in blood donations per 1,000 inhabitants of this age group is much less pronounced than the changes shown in figure $3 \mathrm{~A}$. This indicates that the decrease of blood donations is primarily caused by the demographic change. In women, however, there is an additional decrease of blood donations which adds to the donor loss by the demographic change.

age of RBCs transfused to medical patients is increasing: 2005 37.4\%, 2010 36.4\%, and 2015 39.3\%. However, the absolute number of RBCs transfused to medical patients declined (35,734 RBCs in 2005, 34,616 RBCs in 2010, and 32,430 in 2015). The second largest demand was observed in surgical patients, but in contrast to medical patients both the percentage and the absolute number of transfusions in surgical patients decreased over time (33,530 RBCs (35.1\%) in 2005, 33,249 RBCs (34.9\%) in 2010, and 27,573 RBCs (33.4\%) in 2015). The percentage of RBCs transfused to critically ill or emergency patients increased over the study period, while the absolute number also declined (23,432 RBCs (24.6\%) in 2005, 26,137 RBCs (27.5\%) in 2010, and 21,651 RBCs (26.2\%) in 2015). As shown in figure $5 \mathrm{~A}$ most of the in-hospital RBCs were transfused to patients $\geq 65$ years. This was true for surgical patients (65.1\% of the RBC transfusion demand in this group); medical patients (63.8\% of the RBC transfusion demand in this group), and critically ill / emergency patients $(60.4 \%$ of the RBC transfusion demand in this group) (table 1). The overall reduction of the transfusion demand of 12,864 RBCs from 2005 to 2015 was mostly caused by a reduction of RBC transfusions in surgical patients, which contribute $46.3 \%$ to the overall reduction in transfusion demand. Interestingly the decreasing number of transfusions in surgical patients can mostly be ascribed to patients 65 years and older ( $60.6 \%$ of the reduction in surgical patients). In comparison, the decrease of the RBC transfusion demand in medical patients accounted for only $25.7 \%$ of the overall reduction in RBC demand. However, $75.1 \%$ of the total reduction of the transfusion demand in medical patients also occurred in patients $\geq 65$ years. In contrast, the decline of the RBC transfusion demand in critically ill / emergency patients was mostly achieved among patients younger than 65 years ( $81.1 \%$ of the absolute reduction in this patient group).

This overall decrease in the transfusion demand occurred although there was an increase in the population of age groups $\geq 65$ years in Mecklenburg-Western Pomerania from 19.7\% in 2005 to 

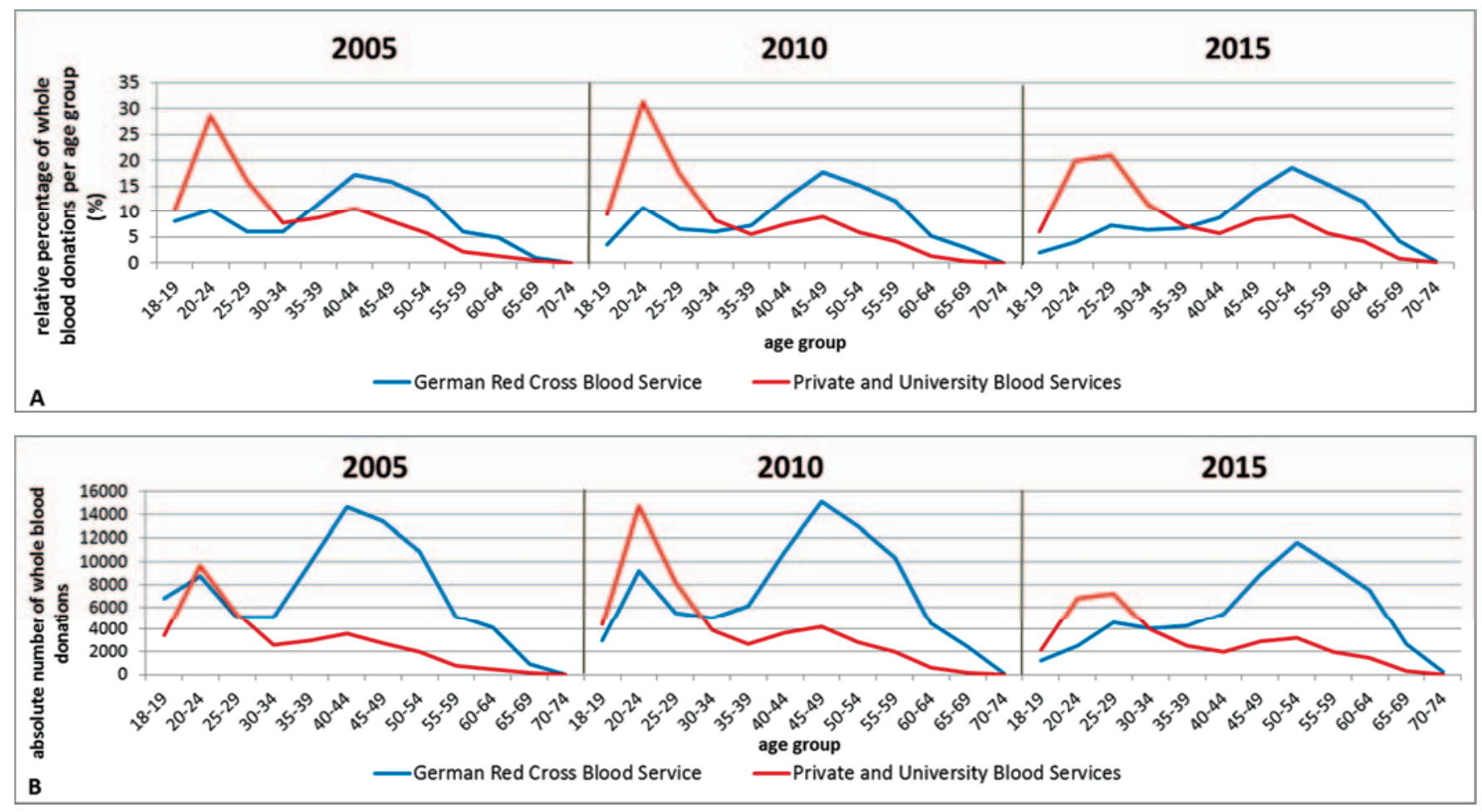

Fig. 4. A Relative percentage of whole blood donations for the German Red Cross Blood Service and University and private blood services (all blood donations of a single blood donation service correspond to $100 \%$ ). The different blood services in the state recruit different patient groups. While the Red Cross blood donation service primarily recruits donors in the age groups $>30$ years, the university-based and the private blood service primarily recruit donors $<30$ years of age. The figure shows the relative proportions of donors per age group of the different blood services. B Absolute number of whole blood donations for the German Red Cross Blood Service and University and private blood services. The Red Cross blood donation service obtains most blood donations in the state. The reduction in absolute donations between 2005 and 2015 is obvious for all blood services in the state.

Table 1. RBC transfusions by patient classification (all absolute numbers refer to the number of transfused RBCs)

\begin{tabular}{|c|c|c|c|c|c|}
\hline & Surgical patients & $\begin{array}{l}\text { Critically ill / } \\
\text { emergency patients }\end{array}$ & Medical patients & Other ${ }^{*}$ & Total \\
\hline \multicolumn{6}{|l|}{$\begin{array}{l}\text { Absolute number of transfusions and percentage } \\
\text { by patient classification }\end{array}$} \\
\hline \multirow[t]{2}{*}{2005} & 33,530 & 23,432 & 35,734 & 2,759 & 95,455 \\
\hline & $35.1 \%$ & $24.6 \%$ & $37.4 \%$ & $2.9 \%$ & \\
\hline \multirow[t]{2}{*}{2010} & 33,249 & 26,137 & 34,616 & 1,198 & 95,200 \\
\hline & $34.9 \%$ & $27.4 \%$ & $36.4 \%$ & $1.3 \%$ & \\
\hline \multirow[t]{2}{*}{2015} & 27,573 & 21,653 & 32,431 & 934 & 82,591 \\
\hline & $33.4 \%$ & $26.2 \%$ & $39.3 \%$ & $1.1 \%$ & \\
\hline \multicolumn{6}{|l|}{ RBCs transfused to patients $\geq 65$ years } \\
\hline \multirow[t]{2}{*}{2005} & 21,549 & 13,417 & 23,172 & 1,178 & 59,316 \\
\hline & $64.3 \%$ & $57.3 \%$ & $64.8 \%$ & $42.7 \%$ & $62.1 \%$ \\
\hline \multirow[t]{2}{*}{2010} & 22,874 & 16,488 & 23,428 & 271 & 63,061 \\
\hline & $68.8 \%$ & $63.1 \%$ & $67.7 \%$ & $22.6 \%$ & $66.2 \%$ \\
\hline \multirow[t]{2}{*}{2015} & 17,941 & 13,078 & 20,693 & 15 & 51,727 \\
\hline & $65.1 \%$ & $60.4 \%$ & $63.8 \%$ & $1.6 \%$ & $62.6 \%$ \\
\hline \multirow{2}{*}{$\begin{array}{l}\text { Absolute reduction of RBC from } 2005 \text { to } 2015 \text { by patient } \\
\text { classification (RBCs of a single patient category in the } \\
\text { year } 2005 \text { correspond to } 100 \% \text { ) }\end{array}$} & $-5,957$ & $-1,779$ & $-3,303$ & $-1,825$ & $-12,864$ \\
\hline & $-17.8 \%$ & $-7.6 \%$ & $-9.2 \%$ & $-66.1 \%$ & $-13.5 \%$ \\
\hline $\begin{array}{l}\text { Amount of RBC transfusion demand reduction according to } \\
\text { patients classification in \% of overall reduction (overall } \\
\text { reduction of } 12,864 \text { RBCs corresponds to } 100 \% \text { ) }\end{array}$ & $-46.3 \%$ & $-13.8 \%$ & $-25.7 \%$ & $-14.2 \%$ & \\
\hline
\end{tabular}



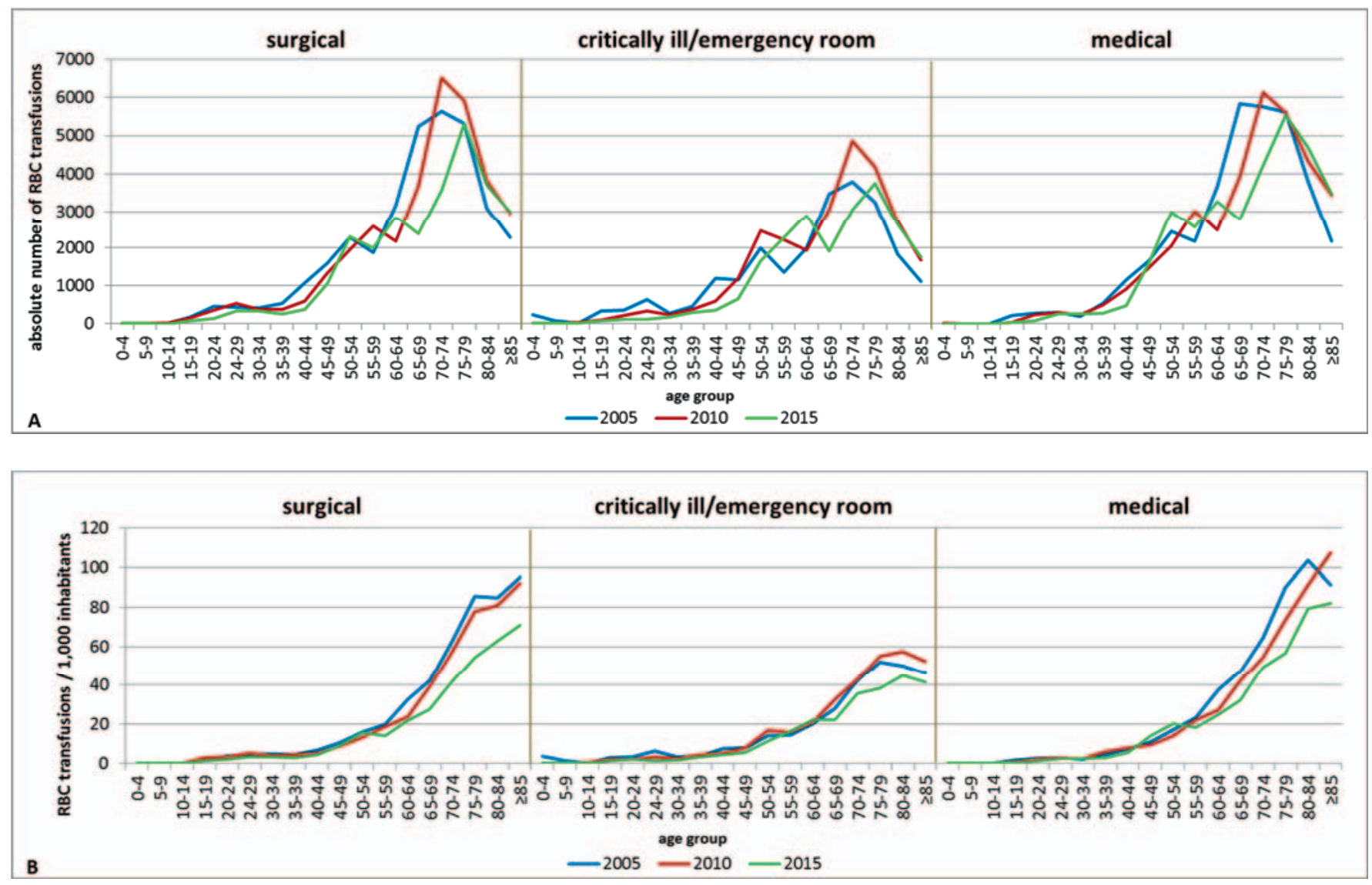

Fig. 5. A Absolute number of RBC transfusions by patient classification. The transfusion demand for in-hospital patients decreased substantially between 2005 and 2015. This accounts for all age groups and all patient groups. B RBC transfusions per 1,000 inhabitants of the respective age group by patient classification. The relative transfusion demand per 1,000 population per age group takes the demographic change into account and clearly shows a substantial change in medical practice leading to reduction of the transfusion demand in all age groups, mostly pronounced in patients older than 60 years.

Table 2. $\mathrm{RBC}$ transfusions and transfusion rate per 1,000 inhabitants per age group

\begin{tabular}{|c|c|c|c|c|c|c|c|c|}
\hline \multirow[t]{2}{*}{ Age group, years } & \multicolumn{4}{|c|}{ Absolute number of RBCs } & \multicolumn{4}{|c|}{ Transfusion rate / 1,000 population } \\
\hline & 2005 & 2010 & 2015 & $\begin{array}{l}\text { \% reduction between } \\
2005 \text { and } 2015\end{array}$ & 2005 & 2010 & 2015 & $\begin{array}{l}\text { \% reduction between } \\
2005 \text { and } 2015\end{array}$ \\
\hline $0-4$ & 452 & 383 & 354 & $-21.7 \%$ & 7.1 & 5.9 & 5.3 & $-25.3 \%$ \\
\hline $5-9$ & 197 & 190 & 221 & $+12.2 \%$ & 3.3 & 3.0 & 3.3 & $+0.6 \%$ \\
\hline $10-14$ & 171 & 161 & 135 & $-21.1 \%$ & 3.2 & 2.7 & 2.1 & $-33.0 \%$ \\
\hline $15-19$ & 902 & 424 & 392 & $-56.5 \%$ & 7.3 & 8.0 & 6.2 & $-14.3 \%$ \\
\hline $20-24$ & 1,096 & 819 & 323 & $-70.5 \%$ & 9.3 & 7.5 & 5.5 & $-40.5 \%$ \\
\hline $24-29$ & 1,398 & 1,173 & 696 & $-50.2 \%$ & 13.8 & 11.5 & 6.7 & $-51.8 \%$ \\
\hline $30-34$ & 924 & 833 & 750 & $-18.8 \%$ & 10.7 & 8.8 & 7.4 & $-30.6 \%$ \\
\hline $35-39$ & 1,556 & 1,219 & 824 & $-47.0 \%$ & 12.8 & 14.4 & 8.7 & $-32.3 \%$ \\
\hline $40-44$ & 3,463 & 2,101 & 1,192 & $-65.6 \%$ & 22.0 & 17.6 & 14.1 & $-36.1 \%$ \\
\hline $45-49$ & 4,508 & 4,006 & 3,272 & $-27.4 \%$ & 29.9 & 26.0 & 28.4 & $-5.1 \%$ \\
\hline 50-54 & 6,861 & 6,445 & 6,930 & $+1.0 \%$ & 48.6 & 43.9 & 46.7 & $-3.9 \%$ \\
\hline 55-59 & 5,572 & 7,775 & 6,796 & $+22.0 \%$ & 59.1 & 56.6 & 48.2 & $-18.5 \%$ \\
\hline 60-64 & 9,039 & 6,610 & 8,970 & $-0.8 \%$ & 92.1 & 72.1 & 68.3 & $-25.3 \%$ \\
\hline 65-69 & 14,715 & 10,686 & 7,063 & $-52.0 \%$ & 118.6 & 114.0 & 81.5 & $-31.3 \%$ \\
\hline 70-74 & 15,597 & 17,570 & 10,836 & $-30.5 \%$ & 174.3 & 155.0 & 126.8 & $-27.3 \%$ \\
\hline 75-79 & 14,560 & 15,836 & 14,624 & $+0.4 \%$ & 234.0 & 208.0 & 149.4 & $-36.2 \%$ \\
\hline $80-84$ & 8,818 & 10,900 & 11,005 & $+20.8 \%$ & 241.9 & 230.2 & 186.8 & $-22.8 \%$ \\
\hline$\geq 85$ & 5,626 & 8,069 & 8,199 & $+45.7 \%$ & 236.3 & 252.8 & 194.7 & $-17.6 \%$ \\
\hline total & 95,455 & 95,200 & $82,582^{*}$ & $-13.5 \%$ & 55.9 & 58.0 & 51.2 & $-8.4 \%$ \\
\hline
\end{tabular}


$23.0 \%$ in 2015 [12]. As the absolute numbers do not take into account the population numbers in different age groups, we analyzed the transfusion rates per 1,000 inhabitants per age group (fig. 5B; table 2). This shows that the biggest reduction in transfusion rates was achieved in the age groups $20-45$ and $65-80$ years and underscores the importance to analyze transfusion rates per 1,000 population per age group rather than total transfusion numbers.

\section{Type of Data Documentation}

In $201570 \%$ of all hospitals completely retrieved their data on $\mathrm{RBC}$ recipients from the hospital IT system, $12 \%$ of the data were partially digital (data were retrieved from laboratory books as well as from the hospital IT system), and $18 \%$ of the hospitals entirely documented non-digital data. As smaller hospitals transfuse less RBCs than larger hospitals, the data of $86 \%$ of all in-hospital RBC transfusions were obtained digitally (70,694 RBCs), $8 \%$ partially digitally $(6,913 \mathrm{RBCs})$, and only $6 \%$ from non-digital sources (4,984 RBCs).

\section{Discussion}

The demographic changes in Mecklenburg-Western Pomerania are typical for the changes in all Eastern federal states of Germany, while in the Western federal states of Germany these changes will manifest about 10 years later. The decline in donation rates was more pronounced in female than in male blood donors. For the transfusion demand, we found that the reduction in the absolute RBC transfusion demand was mostly attributed to decreased transfusion rates in surgical and medical patients older than 60 years.

Beside the decline in birth rate after 1990, a second major change in demography will have an impact on the blood supply. The baby-boomer generation (born 1955-1969) currently provides the largest proportion of all blood donations. With the age shift of the baby boomer generation, the age group over 30 years with most blood donations moved from $40-44$ years in 2005 , to $45-49$ years in 2010, and to 50-54 years in 2015. This strongly indicates that a further major decline in blood donations should be expected in about $10-15$ years, when the baby boomer generation will leave the donor pool because of age or increasing comorbidities. The more liberal regulations in regard to the age limit of blood donors will only partially compensate for this loss in blood donors.

In contrast to the absolute decrease of whole blood donations, the decline in donation rates per 1,000 inhabitants was less pronounced, especially when comparing the years 2005 and 2015. Interestingly, distinct differences can be observed between both sexes. While donation rates of male donors remained rather constant between 2005 and 2015, donation rates of female donors decreased by $12.4 \%$. Considering the age group of donors younger than 30 years, the donation rate of men remained stable (2005 vs. 2015), while the donation rates of young females declined by $22.4 \%$ from 2005 to 2015. Thus, the absolute reduction of whole blood donations by male donors seems mostly to be caused by the absolute reduction of the population in the respective age groups, while there is an overproportional loss of female donors. We conclude that we not only loose young female donors due to the demographic change but also a smaller proportion of young women donated blood in 2015 compared to 2005. This loss of young female blood donors needs to be addressed by future donor motivation campaigns. Jóhannsdóttir et al. [16] reported for Iceland that the proportion of female donors could successfully be increased by donor motivation campaigns of the Icelandic Blood Bank especially for women. However, in comparison to Mecklenburg-Western Pomerania women are still strongly underrepresented among the Icelandic blood donor population with $26.7 \%$.

Our study provides evidence that a certain diversity of blood donation services probably is advantageous for securing the blood supply. While the German Red Cross blood donation service in the study region primarily motivated blood donors in the age over 30 years of age, the other blood services were more able to address blood donors below 30 years of age.

The demographic changes will affect all regions in Germany with about 10-year time difference between the federal states in Eastern and Western Germany and a more pronounced change in Eastern Germany due to the decline in birth rate and the migration of young adults after 1990. While in Mecklenburg-Western Pomerania the four blood donation services enhanced their donor recruitment activities during the last 10 years, in Western federal states of Germany donor recruitment activities of several blood donation services are currently reduced to adjust the donation numbers to the decreasing transfusion rates to minimize wastage (personal communication of the author AG with several blood services). As shown in figure 2 , a similar ratio between the potential donor population and people $\geq 65$ years as the 2.71 in Mecklenburg-Western Pomerania in 2015 will be reached 10 years later in 2025 in the Western federal states. If the blood services in the Western federal states now intentionally reduce their donor pool, it could become difficult to reverse the trend of decreasing donation numbers, when similar age structures are reached as currently in the Eastern German federal states. As shown in our study, even the additional activities of a new blood service only transiently counteracted the trend of declining whole blood donations. Currently it would be reasonable to reduce donation rates in elderly donors in the Western German federal states to minimize the risk of blood wastage, but to maintain recruitment of young first-time donors. However, this is extremely difficult to achieve as all motivation campaigns for blood donation will likely motivate primarily long-time donors.

When the baby boomer generation will retire from the active donor pool, the proportion of potential recipients of blood products will increase. Of all RBCs, more than $60 \%$ were transfused to patients older than 65 years. In 2015, this population group already accounted for $23.0 \%$ of the whole population in MecklenburgWestern Pomerania, but it will further increase to about $32 \%$ in $2030[12,15]$. The key question is whether this will lead to an increased transfusion demand or whether the decline in transfusion demand observed over the previous 5 years in Germany will further continue, hereby compensating for the increasing population group older than 65 years. 
Our study has several limitations. The European Directorate for the Quality of Medicines and Health Care reported RBC transfusion rates of 27/1,000 population for the Netherlands and 35/1,000 for Switzerland in 2013, which is much lower than the current transfusion rates in Germany (55/1,000 population) [6]. The first limitation is that due to lack of data, it is currently unclear whether and where the nadir of blood demand has already been reached in Germany. A second limitation is that our study region differs considerably from the densely populated, industrialized, urban areas in Germany. Only sparse information exists whether and how donor behavior differs between those areas.

In times of sensible changes within the donor and recipient populations, data of whole blood donations and transfusion demand should be monitored regularly. For strategic planning as well as for benchmarking with other medical systems, total donation numbers and transfusion data without the additional information on donor and patient age, gender, and underlying disease are not very helpful. As underscored by the present study, donation rates and transfusion demand differ considerably depending on age groups, underlying disease, and gender. In addition, the transfusion demand is strongly influenced by medical practice. Considering the uncertain predictions of demand, as shown by our study, planning the future blood supply requires regular monitoring of blood donation and transfusion demand data. It would be highly desirable to collect these data on a regional basis to identify differences between different regions of Germany. The data of blood donors are already collected and analyzed by the Paul Ehrlich Insti- tute and the Robert Koch Institute on an annual basis for Germany. However, no data exist in regard to the characteristics of transfusion recipients. Unfortunately, in 2015 only $70 \%$ of the participating hospitals of our study obtained their data digitally. In these hospitals, $86 \%$ of all RBCs were transfused. The data on patient characteristics of these transfusions could be provided without the need of additional documentation efforts. This would already be helpful for major strategic decisions of blood services to maintain a sufficient future blood supply, which is one of the major challenges of transfusion safety of the next decades.

\section{Acknowledgments}

We thank Dr. Doris Gloger, Haema Blutspendezentrum Rostock, Rostock, Germany, and Dr. Kirstin Stüpmann, Blutspendedienst Deutsches Rotes Kreuz Mecklenburg-Vorpommern, Rostock, Germany, for providing the data of their blood donation services.

We thank all hospitals in Mecklenburg-Western Pomerania for providing their data on patients' blood transfusions.

\section{Disclosure Statement}

All authors declare that there are no conflicts of interests to declare.

In the study exclusively anonymous data were used. The institutional ethics review board of the Universitätsmedizin Greifswald approved the study.

All authors had full access to all data including all statistical reports and tables used in the manuscript.

\section{References}

1 Greinacher A, Fendrich K, Brzenska R, Kiefel V, Hoffmann W: Implications of demographics on future blood supply: a population-based cross-sectional study. Transfusion 2011;51:702-709.

2 Greinacher A, Weitmann K, Lebsa A, Alpen U, Gloger D, Stangenberg W, Kiefel V, Hoffmann W: A population-based longitudinal study on the implications of demographics on future blood supply. Transfusion 2016;56:2986-2994.

3 Greinacher A, Weitmann K, Schönborn L, Alpen U, Gloger D, Stangenberg W, Stüpmann K, Greger N, Kiefel V, Hoffmann W: A population based longitudinal study on the implication of demographic changes on blood donation and transfusion demand. Blood Advances 2017 1:867-874.

4 Borkent-Raven BA, Janssen MP, van der Poel CL: Demographic changes and predicting blood supply and demand in the Netherlands. Transfusion 2010;50: 2455-2460.

5 Tinegate H, Pendry K, Murphy M, Babra P, Grant-Casey J, Hopkinson C, Hyare J, Rowley M, Seeney F, Watson $\mathrm{D}$, Wallis J: Where do all the red blood cells (RBCs) go? Results of a survey of RBC use in England and North Wales in 2014. Transfusion 2016;56:139-145.

6 Janssen MP, van Hoeven LR, Rautmann G: Trends and Observations on the Collection, Testing and Use of Blood and Blood Components in Europe. 2001-2011 report. www.edqm.eu/medias/fichiers/the_collection_ testing_and_use_of_blood_and_blood_components_in europe_2011_report.pdf (last accessed July 17, 2017).
7 Tinegate H, Chattree S, Iqbal A, Plews D, Whitehead J, Wallis JP: Ten-year pattern of red blood cell use in the North of England. Transfusion 2013;53:483-489.

8 van Hoeven L, Koopman R, Koffijberg H, Roes K, Janssen M: Historical time trends in red blood cell usage in the Netherlands. IJCTM 2016;4:67-77.

9 Volken T, Buser A, Castelli D, Fontana S, Frey BM, Rusges-Wolter I, Sarraj A, Sigle J, Thierbach J, Weingand T, Taleghani BM: Red blood cell use in Switzerland: trends and demographic challenges. Blood Transfus 2016; DOI 10.2450/2016.0079-16.

10 Carson JL, Stanworth SJ, Roubinian N, Fergusson DA, Triulzi D, Doree C, Hebert PC: Transfusion thresholds and other strategies for guiding allogeneic red blood cell transfusion. Cochrane Database Syst Rev 2016;10: CD002042.

11 Sixty-third World Health Assembly: WHA63.12 Availability, Safety and Quality of Blood Products 2010. http://apps.who.int/medicinedocs/documents/s19998en/ s19998en.pdf (last accessed July 17, 2017).

12 Statistisches Bundesamt: Bevölkerung: Bundesländer, Stichtag, Geschlecht, Altersjahre, Wiesbaden, Statistisches Bundesamt, 2017. /www-genesis.destatis.de/genesis/ online/data;jsessionid $=54 F F E 50 E 1230826$ B0196D310F 6DD646D.tomcat_GO_1_1?operation $=$ abruftabelleBea rbeitenelevelindex $=2 \&$ levelid $=1486816342782 \mho$ auswa hloperation=abruftabelleAuspraegungAuswaehlenઐaus wahlverzeichnis=ordnungsstruktur\&auswahlziel $=$ werte abrufeselectionname $=12411-0012 \& a u s w a h l t e x t$ $=\% 23 S D L A N D-13 \% 23 Z-31.12 .2015 \% 2 C 31.12 .2010 \%$ 2C31.12.2005\&werteabruf $=$ Werteabruf (last accessed July 17, 2017).

13 Statistisches Bundesamt: Publikation - Bevölkerung Bevölkerungsentwicklung in den Bundesländern bis 2060 - Statistisches Bundesamt (Destatis). Wiesbaden, Statistisches Bundesamt, 2015. www.destatis.de/ DE/Publikationen/Thematisch/Bevoelkerung/VorausberechnungBevoelkerung/BevoelkerungBundeslaender 2060.html (last accessed July 17, 2017).

14 Statistisches Bundesamt: Statistisches Bundesamt Deutschland - GENESIS-Online. Wiesbaden, Statistisches Bundesamt, 2017. www-genesis.destatis.de/genesis/ online $/$ data $;$ jessionid $=5$ E65E2424BDC01A2E54B574C 54C336F1.tomcat_GO_1_1?operation $=$ ergebnistabelle Umfang\&levelindex $=3 \&$ levelid $=1486815164546 \&$ down loadname $=12411-0011$ (last accessed July 17, 2017).

15 Statistisches Amt Mecklenburg-Vorpommern: A1832 Aktualisierte 4. Landesprognose (Basisjahr 2010): Bevölkerungsentwicklung des Landes sowie der kreisfreien Städte und Landkreise bis 2030 nach Einzelalter 2013.

16 Johannsdottir V, Gudmundsson S, Moller E, Aspelund $\mathrm{T}$, Zoega H: Blood donors in Iceland: a nationwide population-based study from 2005 to 2013 . Transfusion 2016;56:1654-1661. 